\title{
A new method for Rayleigh wave inversion by geological unit merging
}

\author{
Shu Wang ${ }^{1,2}$, and Dengke $\mathrm{He}^{1,2, *}$ \\ ${ }^{1}$ College of Geoscience and Surveying Engineering,China University of Mining and Technology, Beijing, P. R. China \\ ${ }^{2}$ China State Key Laboratory of Coal Resources and Safe Mining, China University of Mining and Technology, Beijing, P. R. China
}

\begin{abstract}
Rayleigh wave exploration is widely used in engineering investigation due to the advantages of speediness, small attenuation, and strong anti-interference ability. However, it is difficult to get accurate results since the Rayleigh wave inversion is a multi-parameter and high nonlinear problem. During the inversion, the strata over the target layer must be divided into many thin layers. The more thin layers are divided, the more parameters are needed to invert. Usually, the number of parameters is much larger than the actual data points, which makes the inversion an under-determined equation. Actually, the several adjacent thin layers will be the parts of a same geological unit. The paper therefore proposed a new method for Rayleigh wave inverting based on geological unit merging into a super unit. Simultaneously, the Bayesian estimation was introduced to determine if the adjacent thin layers could be merged into larger ones. The synthetic model testing demonstrated that the new method is feasible and can effectively improve the accuracy of the inversion.
\end{abstract}

\section{Introduction}

The Rayleigh wave is formed by the interference from $\mathrm{P}$-wave and S-wave near the free surface and has the characteristics of strong energy and weak amplitude attenuation. There will occur an especial dispersion phenomenon when it propagates in the layered media ${ }^{[1-3]}$. Consequently, the especial dispersion is an important inversion tool of geophysical prospecting, which is widely used in the research of near surface structure, continental internal structure, subgrade quality detection, etc $^{[4-6]}$.

There are several methods to calculate the dispersion curve of surface wave in horizontal layered media quickly and accurately, like Thomson Haskell algorithm ${ }^{[7-8]}$, Knopoff algorithm ${ }^{[9-10]}$, Abo-Zena algorithm ${ }^{[11]}$, reflection and transmission matrix algorithm $^{[12-14]}$, which promoted surface wave exploration.

During the exploration, the strata over the target layer must be divided into many thin layers according to the finite element method for simulating calculation ${ }^{[15]}$. The extensive thin layers will lead to large amount of unknown parameters to be calculated, which will usually make the objective function into an under-determined equation. In order to solve the problem, this paper proposes a new method based on geological unit merging. The several adjacent thin layers can be merged into a larger one when their geophysical parameters belong to the predetermined threshold based on Bayesian estimation.

\section{Rayleigh wave inversion}

The phase velocity of Rayleigh wave can be expressed by a nonlinear implicit function of stratum characteristic parameters ${ }^{[16]}$.

$$
F\left(\mathbf{V}_{\mathbf{R} j}, f_{j}, \mathbf{V}_{\mathbf{S}}, \mathbf{V}_{\mathbf{p}}, \boldsymbol{\rho}, \mathbf{H}\right)=0
$$

where, $f_{j}$ is the $j$ th frequency element $(j=1,2, \cdots, m) ; \quad \mathbf{V}_{\mathbf{R} j}$ is the phase velocity at the frequency $f_{j} ; \mathbf{V}_{\mathbf{s}}=\left(V_{s 1}, V_{s 2}, \cdots, V_{s n}\right)^{\mathrm{T}}$, $\mathbf{V}_{\mathbf{P}}=\left(V_{p 1}, V_{p 2}, \cdots, V_{p n}\right)^{\mathrm{T}}, \boldsymbol{\rho}=\left(\rho_{1}, \rho_{2}, \cdots, \rho_{n}\right)^{\mathrm{T}}$ and $\mathbf{H}=\left(H_{1}, H_{2}, \cdots, H_{n}\right)^{\mathrm{T}}$ are the vectors respectively represented the S-wave velocity, the P-wave velocity, the density and the thickness about the $n$ layers of geological model.

The P-wave velocity and density can be represented by $\mathrm{S}$-wave velocity according to Poisson's ratio and Gardner ${ }^{[17]}$ since the dispersion equation is not sensitive to them. The total thickness over the target layer can be divided into enough number of thin layers with equal thickness according to the finite element method. Finally, the unknown parameter in the Eq.(1) can be simplified as only S-wave velocity, and Eq.(1) can be abbreviated as

$$
\mathbf{V}_{\mathbf{R} j}=F\left(\mathbf{V}_{\mathbf{S}}, f_{j}\right)
$$


The Rayleigh wave inversion based on the dispersion curve can be implemented by the Eq.(2) above.

\section{A new method of Rayleigh wave inversion based on geological unit merging}

How to divide the whole geological structure over the deepest target layer is a significant problem in the current Rayleigh wave inversion. The more thin layers the strata are divided into, the better depiction the wave impedance interfaces are described by, however, the more unknown parameters are needed to invert and more than the number of acquired seismic traces. The inversion may fall into local extremum just for getting a better solution in accordance with the objective function, thus reducing the inversion accuracy.

In order to solve this problem, the paper proposes a new method based on geological unit merging through analyzing the parameters' iterating tendencies in the inverted process, in which the parameters and their iterating tendencies of the adjacent thin layers are similar when these thin layers are split from the same stratum. Consequently, the adjacent thin layers can be emerged to a larger one, which will decrease the total number of the parameters needed to invert and improve the inversion accuracy.

After the $k^{\text {th }}$ iteration, if the shear wave velocities of the two adjacent layers are similar, they will have the same geological parameters, represented by Eq.(3).

$$
\mathbf{V}_{\mathbf{S} l+1}^{k+1}=\mathbf{V}_{\mathbf{S} l}^{k+1}=\frac{1}{2}\left(\mathbf{V}_{\mathbf{S} l+1}^{k}+\mathbf{V}_{\mathbf{S} l}^{k}\right)
$$

The layers noted by $l^{\text {th }}$ and $l+l^{\text {th }}$ will update together in the later iteration. The algorithm is feasible under the theoretical noiseless hypothesis, however, the inverted results would be disturbed by random noises of the actual data acquired in industrial exploration. The new method adopts Bayesian theory to estimate the probability whether the adjacent thin layers can be emerged according to the prior probability calculated by the background noise.

$$
\begin{aligned}
& P\left(\mathbf{V}_{\mathbf{S}} \mid \mathbf{V}_{\mathbf{R}}\right)=\frac{P\left(\mathbf{V}_{\mathbf{S}}\right) \cdot P\left(\mathbf{V}_{\mathbf{R}} \mid \mathbf{V}_{\mathbf{S}}\right)}{P\left(\mathbf{V}_{\mathbf{R}}\right)} \\
& \chi^{2}=\mathbf{r}^{\mathbf{T}} \mathbf{C}_{\mathbf{V}_{\mathbf{R}}}^{-1} \mathbf{r}=\sum_{i=1}^{n} \frac{\left(\mathbf{V}_{\mathbf{R} i}-\mathbf{G}_{i} \mathbf{V}_{\mathbf{S} i}\right)^{2}}{\sigma_{i}^{2}}
\end{aligned}
$$

Where, $\mathbf{G}$ is the stiffness matrix, $\mathbf{C}_{\mathbf{V}_{\mathbf{R}}}^{-1}$ is the covariance matrix of the data represented, which is the noise level. The posterior distribution of noise represented as chi square distribution can determine if merging under the given threshold.

\section{Numerical simulation testing}

A 4-layer geological model with a stiff layer sandwiched between two soft layers was chosen to test the new method here. This model could simulate shallow engineering investigations. Because the density and P-wave velocity are not sensitive to the inversion of Rayleigh wave, the density was uniformly set as $1.8 \mathrm{~g} / \mathrm{cm}^{3}$, and the velocity of P-wave was calculated according to Poisson's ratio (as shown in Table 1). Simultaneously, $10 \%$ noise was added into the dispersion curve for testing Bayesian estimation. Fig. 1a and Fig. 1b show the S-wave velocity structure and the basic mode of dispersion curve obtained by forward modeling, respectively.

Table 1. 4-layer model parameters

\begin{tabular}{|l|l|l|l|l|}
\hline $\begin{array}{l}\text { Layer } \\
\text { number }\end{array}$ & $\begin{array}{c}V s \text { velocity } \\
(\mathrm{m} / \mathrm{s})\end{array}$ & $\begin{array}{c}V p \text { velocity } \\
(\mathrm{m} / \mathrm{s})\end{array}$ & $\begin{array}{c}\text { Density } \\
\left(\mathrm{g} / \mathrm{cm}^{3}\right)\end{array}$ & $\begin{array}{c}\text { Thickness } \\
(\mathrm{m})\end{array}$ \\
\hline 1 & 120 & 323 & 1.8 & 10 \\
\hline 2 & 250 & 612 & 1.8 & 5 \\
\hdashline 3 & 200 & 663 & 1.8 & 10 \\
\hdashline 4 & 400 & 980 & 1.8 & $\infty$ \\
\hline
\end{tabular}

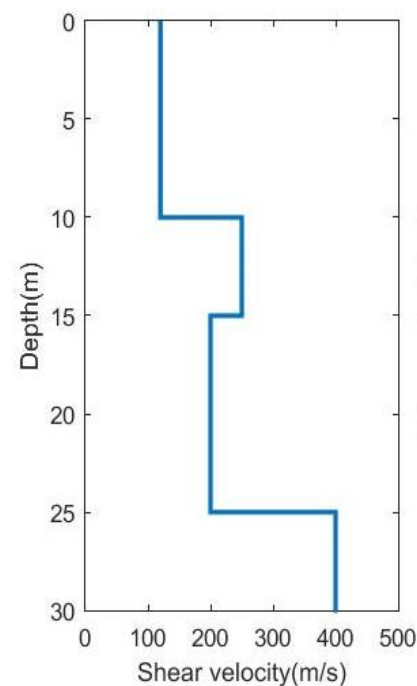

(a)

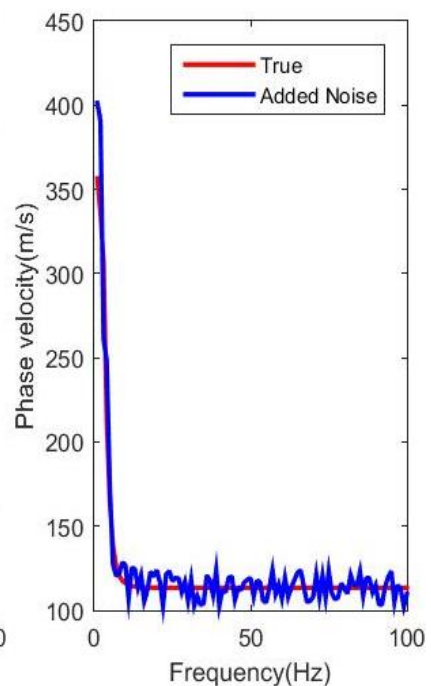

(b)
Fig. 1. The 4-layer model. (a) S-wave velocity structure; (b) the dispersion curve 


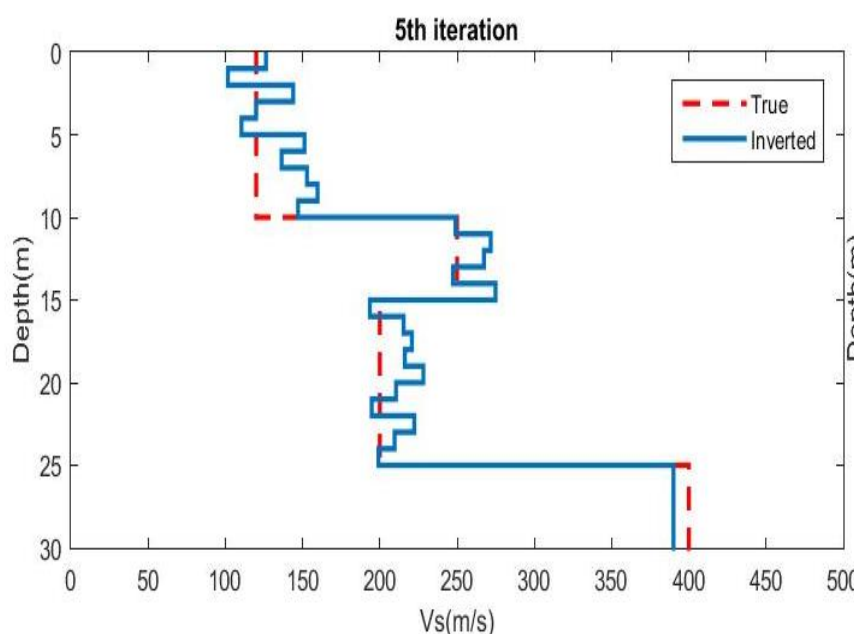

(a)

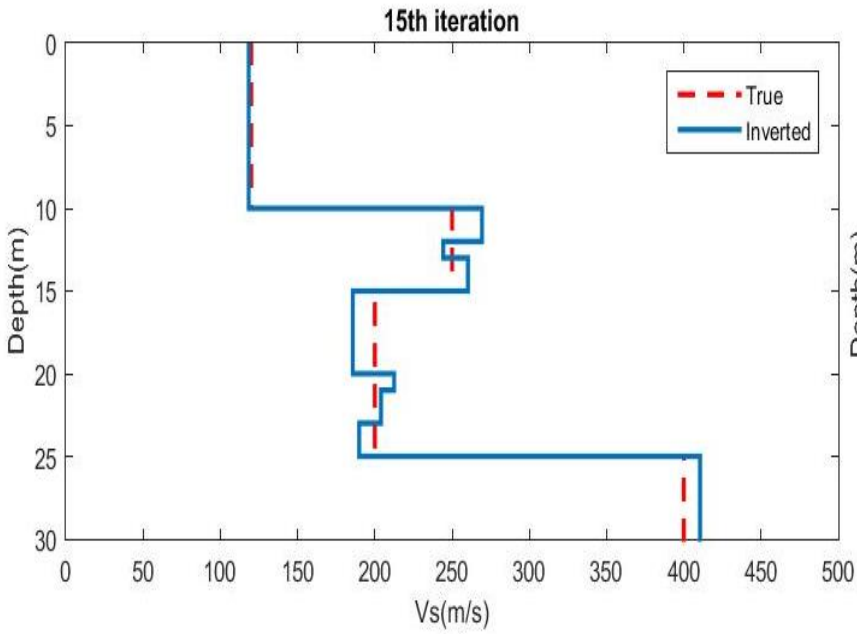

(c)

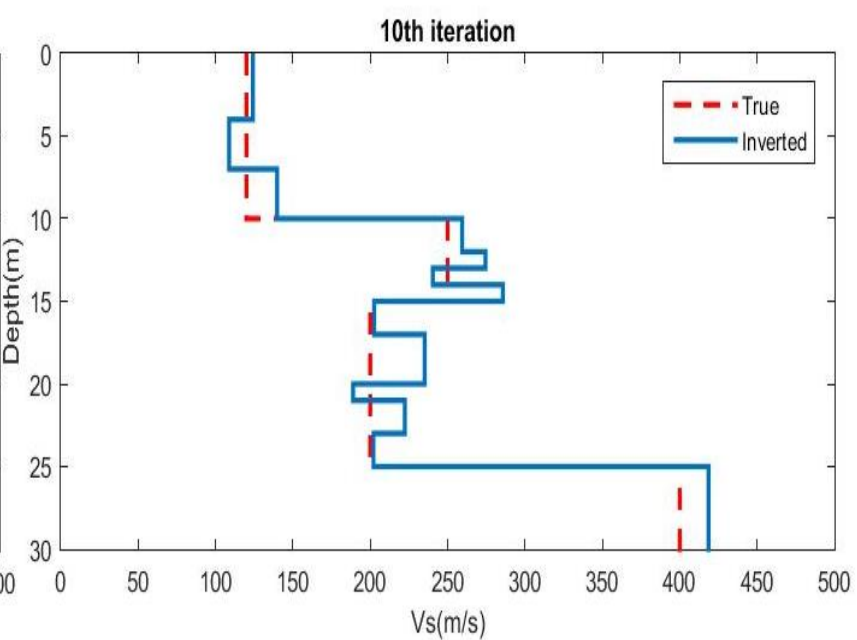

(b)

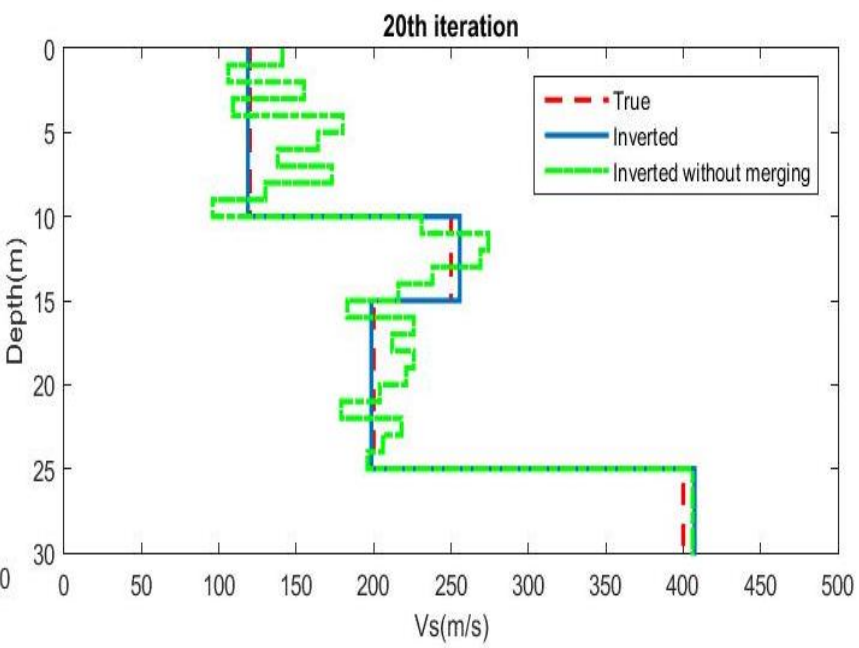

(d)

Fig. 2. The merging process during the inversion of the new method. (a) the $5^{\text {th }}$ iteration; (b) the $10^{\text {th }}$ iteration; (c) the $15^{\text {th }}$ iteration;

(d) the $20^{\text {th }}$ iteration and the result inverted without merging.

The initial model was divided as 25 layers with $1 \mathrm{~m}$ thickness. The differences between the results about the first few iterations and the true model was obvious, since the noise aggravates the instability of inversion. Therefore, to estimate and merge the geological thin layers was begun from the stable time about the inversion tendency, like the $5^{\text {th }}$ iteration here shown in the Fig. 2a. After the 10th iteration shown as the Fig 2b, the model was merged into a 13-layer model. After the $15^{\text {th }}$ iteration, the shallow layer was basically merged, but there was still a large error in the deep part. Finally, when it was the $20^{\text {th }}$ iteration, the inverted results were almost the same as the actual model, and the relative difference was about $1.46 \%$. By contrast, the relative error from the conventional inversion was $13.99 \%$, and the stratification boundary was not clear enough shown as the Fig. $2 \mathrm{~d}$. The inversion test above demonstrates the new method can be feasible and improve the prospecting accuracy.

\section{Conclusion}

Rayleigh wave dispersion curve inversion is a geophysical inversion problem with multi-parameters, and high non-linearity. There is an under-determined problem in the common inversion by the finite element method. To focus on its inversion accuracy and stability, this paper proposed a new method based geological unit merging. The initial model can be divided as enough thin layers according to prospecting demand. When the inversion tendency is stable, the new method will estimate the adjacent thin layers if they should from the same geological stratum based on the Bayesian probability theory. The total number of thin layers will decrease largely after merging the adjacent thin layers, therefore the inverted results will be more stable and accurate. The theoretical inversion testing of the paper demonstrates that the new method is not only feasible but also can effectively improve the inversion accuracy.

\section{Acknowledgment}

The authors thank these following projects for financial supporting this research, including the Fundamental Research Funds for the Central Universities (Grant No. 2011QD04), and Yue Qi Distinguished Scholar Project, China University of Mining \& Technology, Beijing (2019JCA01). 


\section{Reference}

1. L. Rayleigh, P. Lond. Math. Soc. 17, (1887)

2. C.B. Park, R.D. Miller, J. Xia, Geophysics, 64, (1999)

3. J. Xia, R.D. Miller, C.B. Park, Geophysics, 64,

4. J.T. Pan, Y.H. Li, Q.J. Wu, et al, Chin. J. Geophys., 57, (2014)

5. Z.X. Huang, H.Y. Li, Y. Xu, Chin. J. Geophys., 57, (2014)

6. J.P. Liu, Y.H. Luo, W.B. He, Chin. J. Geotech. Eng., 31, (2009)

7. W.T. Thomson, J. Appl. Phys., 21, (1950)

8. N.A. Haskell, B. Seismol. Soc. Am., 43, (1953)

9. L. Knopoff, B. Seismol. Soc. Am.,54, (1964)
10. F.A. Schwab, L. Knopoff, B. Seismol. Soc. Am., 60, (1970)

11. A. Abo-Zena, Geophysical Journal of the Royal Astronomical Society, 58, (1979)

12. B.L. Kennett, B. Seismol. Soc. Am., 64, (1974)

13. X. Chen, Geophys. J. Int., 115, (1993)

14. Y.F. He, W.T. Chen, X.F. Chen, Chin. J. Geophys., 49, (2006)

15. G.Z. Shao, Q.C. Li, J. Earth Sci. Environ., 33, (2011)

16. S. Nazarian, Ph.D. Dissertation, University of Texas, Austin. (1984)

17. G.H.F. Gardner, L.W. Gardner, and A.R. Gregory, Geophysics, 39, (1974) 